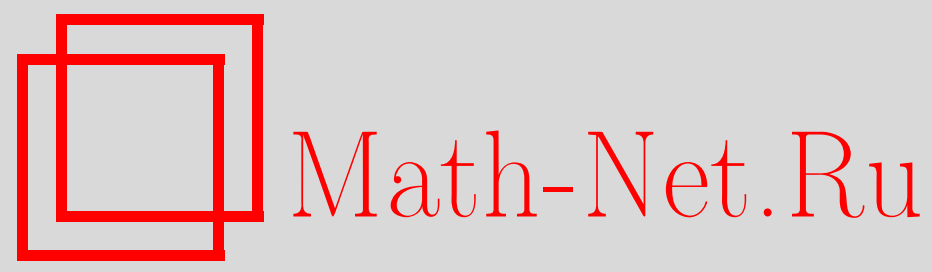

А. М. Чеботарев, Х. К. Гарсиа, Р. Б. Гезада, Об уравнении Линдблада с неограниченными переменными коэффициентами, Матем. заметки, 1997, том 61, выпуск 1, 125-140

DOI: https://doi.org/10.4213/mzm1487

Использование Общероссийского математического портала Math-Net.Ru подразумевает, что вы прочитали и согласны с пользовательским соглашением http://www.mathnet.ru/rus/agreement

Параметры загрузки:

IP : 54.198 .187 .58

26 апреля 2023 г., 03:03:16

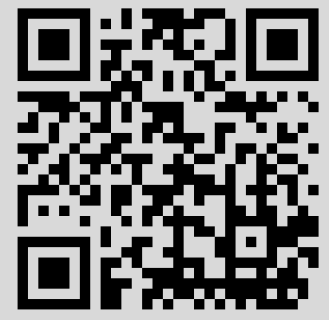




\title{
ОБ УРАВНЕНИИ ЛИНДБЛАДА С НЕОГРАНИЧЕННЫМИ ПЕРЕМЕННЫМИ КОЭФФИЦИЕНТАМИ
}

\author{
А. М. Чеботарев, Х. К. Гарсия, Р.Б. Гезада
}

\begin{abstract}
Получены новые априорные оценки для резольвент минимальных квантовых динамических полугрупп, которые позволяют упростить известные достаточные условия консервативности и ввести условия непрерывности операторных коэффициентов, зависящих от времени, гарантирующие существование консервативных решений уравнений марковской эволюции.

Библиография: 11 названий.
\end{abstract}

В статье вводится операторозначное условие непрерьвности для инфинитезимальных операторов уравнения марковской эволюции, описывающих динамику открытых квантовых систем в алгебре ограниченных операторов $\mathscr{B}(\mathscr{H})$ и в алгебре операторов с конечным следом $\mathscr{T}(\mathscr{H})$ над гильбертовьг пространством $\mathscr{H}$ (см. [1]-[3]). Сжимающая полугрупша $P_{t}(\cdot)$, действуюшая в $\mathscr{B}(\mathscr{H})$ и обладающая свойствами вполне положительности, нормальности, консервативности и ультраслабой непрерьвности, называется квантовой динамической полугруппой [3]. Свойства нормальности и ультраслабой непрерьвности означают соответственно, что l.u.b. $P_{t}\left(X_{n}\right)=P_{t}\left(\right.$ l.u.b. $\left.X_{n}\right)$ и

$$
\lim _{t \rightarrow 0} \operatorname{Tr}\left\{\rho\left(P_{t}(B)-B\right)\right\}=0
$$

для любых операторов $\rho \in \mathscr{T}(\mathscr{H}), B \in \mathscr{B}(\mathscr{H})$. Свойство консервативности в гейзенберговском представлении означает сохранение единицы $I$ алгебры наблюдаемых $\mathscr{B}(\mathscr{H})$, т.е. $P_{t}(I) \equiv I \forall t \geqslant 0$, а в шрёдингеровском представлении - сохранение следа начального состояния $\rho \in \mathscr{T}(\mathscr{H})$ в процессе эволюции: $\operatorname{Tr} T_{t}(\rho) \equiv \operatorname{Tr} \rho \forall t \geqslant 0$, где $T_{t}: \mathscr{T}(\mathscr{H}) \rightarrow \mathscr{T}(\mathscr{H})$ и $P_{t}: \mathscr{B}(\mathscr{H}) \rightarrow \mathscr{B}(\mathscr{H})$ - двойственные полугруппы: $\operatorname{Tr}\left\{T_{t}(\rho) B\right\}=$

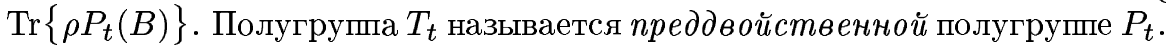

В работах [4]-[6] было показано, что при естественных предположениях об операторных коэффициентах формального генератора $\mathscr{L}(\cdot)$ существует минимальная квантовая динамическая полугруппа $P_{t}^{\min }(\cdot)=\exp \{t \mathscr{L}(\cdot)\}$, консервативность которой является необходимьм и достаточным условием отсутствия других консервативных квантовых динамических полугрупп с тем же формальньм генератором. Поэтому генераторы консервативных минимальных полугрупп называются регулярными.

Работапервого автора вьполнена при поддержке Российского фонда фундаментальных исследований, грант № 95-01-00784. Работа второго и третьего авторов выполнена при поддержке фонда CONACYT Mexico, грант № 0233P-E9506. 
Условие непрерьвности выделяет класс регулярных инфинитезимальньх операторов с коэффициентами, зависящими от времени. В настоящей работе показано, что при подходящем выборе операторозначных условий непрерывности можно гарантировать существование и консервативность решения задачи Коши для уравнения марковской эволюции (уравнения Линдблада) с переменными коэффициентами

$$
\frac{d}{d t} P_{t}(\mathscr{B})=\mathscr{L}_{t}\left(P_{t}(\mathscr{B})\right),\left.\quad P_{t}(\mathscr{B})\right|_{t=0}=B
$$

определяемого формальным инфинитезимальным оператором $\mathscr{L}_{t}(\cdot)$ с переменными коэффициентами

$$
\mathscr{L}_{t}(B)=\Phi_{t}(B)-\Phi_{t}(I) \circ B+i\left[H_{t}, B\right], \quad H_{t}=H_{t}^{*}, \quad \Phi_{t}(\cdot) \in \operatorname{CPn}(\mathscr{H})
$$

где $\operatorname{CPn}(\mathscr{H})$ - конус вполне положительных нормальных отображений алгебры $\mathscr{B}(\mathscr{H})$ (см. [3]). Теория уравнений с переменными коэффициентами играет важную роль при решении задач в представлении взаимодействия.

Статья состоит из пяти разделов. В первом разделе излагается алгебраическая сторона теории, связанная с общими свойствами минимальньх решений резольвентного уравнения [4], [5]. Во втором разделе мы обсуждаем априорные оценки минимальных решений однородного и неоднородного уравнения Линдблада, вытекающие из условия $\mathscr{L}(\Lambda) \leqslant c \Lambda$ для какого-нибудь положительного самосопряженного оператора $\Lambda \geqslant \Phi(I)$, которое обеспечивает единственность решения задачи Коши в классе динамических полугрупп при дополнительных предположениях об областях определения оператора $\Lambda$ и коэффищиентов инфинитезимального оператора $\mathscr{L}(\cdot)$. В третьем разделе получены оценки решения двойственного уравнения в алгебре операторов с конечньм следом. В четвертом разделе вводится условие непрерьвности, позволяющее описать класс инфинитезимальных операторов с переменными коэффициентами как пополнение множества инфинитезимальных операторов $\mathscr{L}_{t}(\cdot)$ с кусочно постоянньми коэффициентами $\Phi_{t}(\cdot), H_{t}$, удовлетворяющими достаточньпм условиям консервативности в каждой точке $t \in \mathbb{R}_{+}$. Основной результат статьи вывод глобального условия консервативности из локальных достаточных условий консервативности. В пятом разделе рассмотрен пример, иллюстрирующий наши основные результаты.

1. Априорные оценки и достаточные условия консервативности. Алгебраическая часть теории. В работе [4] была предложена удобная форма резольвентного уравнения для квантовой динамической полугруппы $P_{t}(\cdot)=\exp \{t \mathscr{L}\}(\cdot)$ на алгебре фон Неймана $\mathscr{B}(\mathscr{H})$ с генератором $\mathscr{L}(B)=\Phi(B)-\Phi(I) \circ B+i[H, B], H=H^{*}$, $\Phi(\cdot) \in \mathrm{CP}(\mathscr{H})$, не зависящим от времени. Резольвентное уравнение имеет вид

$$
R_{\lambda}(B)=\int_{0}^{\infty} d t e^{-\lambda t} P_{t}(B)=A_{\lambda}(B)+Q_{\lambda}\left(R_{\lambda}(B)\right), \quad B \in \mathscr{B}(\mathscr{H})
$$

где $A_{\lambda}(\cdot)$ и $Q_{\lambda}(\cdot)$ - вполне положительные сжимающие отображения,

$$
A_{\lambda}(B)=\int_{0}^{\infty} d t e^{-\lambda t} W_{t}^{*} B W_{t}, \quad Q_{\lambda}(B)=\int_{0}^{\infty} d t e^{-\lambda t} W_{t}^{*} \Phi(B) W_{t},
$$


a $W_{t}=\exp \{-G t\}-$ сильно непрерьвная сжимающая полугрупша в $\mathscr{H}$ с генератором $-G: G \psi=i H \psi+\frac{1}{2} \Phi(I) \psi \forall \psi \in \operatorname{dom} H \cap \operatorname{dom} \Phi(I)$. В [4] (см. также [5], [6]) показано, что ряд $\sum Q_{\lambda}^{k}\left(A_{\lambda}(\cdot)\right)$ сильно сходится на $\mathscr{B}(\mathscr{H})$ к резольвенте минимальной динамической полугруппы

$$
R_{\lambda}^{\min }(B)=\sum_{k=0}^{\infty} Q_{\lambda}^{k}\left(A_{\lambda}(B)\right)
$$

Ее минимальность означает, что разность $R_{\lambda}(\cdot)-R_{\lambda}^{\min }(\cdot) \in \mathrm{CP}(\mathscr{H})$ является вполне положительным отображением для резольвенты $R_{\lambda}(\cdot)$ любой другой динамической полугруппы с тем же формальным генератором $\mathscr{L}(\cdot)$.

Интегрируя по частям интегральное представление (1.2) отображения $A_{\lambda}$, получаем цепочку тождеств

$$
A_{\lambda}(I)+\lambda^{-1} Q_{\lambda}(I) \equiv \lambda^{-1} I, \quad \sum_{k=0}^{n} Q_{\lambda}^{k}\left(A_{\lambda}(I)\right)+\lambda^{-1} Q_{\lambda}^{n+1}(I) \equiv \lambda^{-1} I \quad \forall n>1,
$$

из которых следует

(1) монотонное убьвание последовательности ограниченных положительных операторов $Q_{\lambda}^{n}(I)$

(2) априорная оценка для суммы:

$$
\sum_{k=0}^{n} Q_{\lambda}^{k}\left(A_{\lambda}(I)\right) \leqslant \lambda^{-1} I
$$

(3) представление резольвенты квантовой динамической полугруппы в виде ряда

$$
R_{\lambda}(I)=\sum_{k=0}^{\infty} Q_{\lambda}^{k}\left(A_{\lambda}(I)\right)+\lambda^{-1} \inf _{n} Q_{\lambda}^{n}(I)=R_{\lambda}^{\min }(I)+\lambda^{-1} \inf _{n} Q_{\lambda}^{n}(I) .
$$

Последнее тождество показывает, что $R_{\lambda}^{\min }(I)=\lambda^{-1} I$ и $P_{t}^{\min }(I) \equiv I$, если и только если $\inf _{n} Q_{\lambda}^{n}(I)=0$ для какого-либо $\lambda>0$ (см. [4]). В противном случае существует стационарная точка $X_{\lambda}=\inf _{n} Q_{\lambda}^{n}(I) \geqslant 0$, являющаяся наибольшим собственным оператором отображения $Q_{\lambda}(\cdot)$ в классе положительных операторов с нормой, равной 1 . Если $R_{\lambda}^{\min }(I) \leqslant \lambda^{-1} I$, то квантовая динамическая полугруппа $P_{t}(\cdot)$ с тем же формальным генератором $\mathscr{L}(\cdot)$ консервативна тогда и только тогда, когда $R_{\lambda}(I)-R_{\lambda}^{\min }(I)=X_{\lambda}$.

В случае непрерьвной по норме полугруппы с генератором $\mathscr{L}(\cdot): \mathscr{L}(B)=\Phi(B)-$ $\Phi(I) \circ B+i[H, B]$, неравенство

$$
\mathscr{L}(\Lambda) \leqslant c \Lambda, \quad c \in \mathbb{R}_{+},
$$

для некоторого положительного самосопряженного оператора $\Lambda \in \mathscr{B}(\mathscr{H})$ дает априорные оценки для полугруппы и резольвенты

$$
\begin{aligned}
P_{t}(\Lambda) & =\Lambda+t \mathscr{L}(\Lambda)+\cdots+\frac{t^{n}}{n !} \mathscr{L}^{n}(\Lambda)+\cdots \leqslant \Lambda e^{c t}, \\
R_{\lambda}(\Lambda) & =\int_{0}^{\infty} d t e^{-\lambda t} P_{t}(\Lambda) \leqslant(\lambda-c)^{-1} \Lambda \quad \forall \lambda>c .
\end{aligned}
$$


Рассмотрим банахову подалгебру $\mathscr{T}_{\Lambda}(\mathscr{H})$ алгебры $\mathscr{T}(\mathscr{H})$, порождаемую линейной оболочкой множества положительных операторов $\rho$ с конечным следом таких, что плотно определенный оператор $\rho \Lambda$ допускает ограниченное продолжение на $\mathscr{H}$ и имеет конечньй след. Оценка (1.5) означает, что полугрупп $T_{t}$ оставляет инвариантной подалгебру $\mathscr{T}_{\Lambda}(\mathscr{H})$. Действительно,

$$
\operatorname{Tr}\left\{T_{t}(\rho) \Lambda\right\}=\operatorname{Tr}\left\{\rho P_{t}(\Lambda)\right\} \leqslant e^{c t} \operatorname{Tr} \rho \Lambda,\|\rho\|_{\mathscr{T}_{\lambda}}=\operatorname{Tr} \rho \Lambda \quad \forall \rho \in \mathscr{T}_{\Lambda}^{+}(\mathscr{H}) .
$$

Заметим, что $A_{\lambda}(\Phi(I)) \equiv Q_{\lambda}(I)$. Поэтому если $\Phi(I) \leqslant \Lambda$, то из $(1.5)$ и разложения (1.3) для резольвенты следует оценка для суммы ряда

$$
\sum_{1}^{\infty} Q_{\lambda}^{k}(I)=R_{\lambda}(\Phi(I)) \leqslant R_{\lambda}(\Lambda) \leqslant(\lambda-c)^{-1} \Lambda \quad \forall \lambda>c .
$$

Как мы увидим ниже, эта оценка распространяется на резольвенты минимальных квантовых динамических полугрупп с неограниченными коэффициентами:

$$
\sum_{1}^{\infty} Q_{\lambda}^{k}(I)=R_{\lambda}^{\min }(\Phi(I)) \leqslant(\lambda-c)^{-1} \Lambda \quad \forall \lambda>c .
$$

Следовательно, $Q_{\lambda}^{n}(I) \stackrel{w}{\rightarrow} 0$ при $n \rightarrow \infty$ на плотном в $\mathscr{H}$ множества dom $\Lambda$. Отсюда следует сильная сходимость $Q_{\lambda}^{n}(I) \stackrel{s}{\rightarrow} 0$, и поэтому условие (1.4) является достаточным для консервативности минимальной динамической полугрушы [6]. Априорные оценки (1.5) для минимальной резольвенты и условие (1.4) в случае $\Lambda=\Phi(I)$ были рассмотрены в [7]. Важное наблюдение о том, что в качестве оператора $\Lambda$ можно использовать операторы, мажорирующие $\Phi(I)$, впервые было сделано в работе [8].

Этот результат очевидным образом распространяется на полугруппы с кусочно постоянньми производящими операторами, удовлетворяющими в каждой точке условию (1.4). Оказьвается, что оно остается справедливьм и для производящих операторов, зависящих от времени и непрерьвных в следующем смысле:

$$
\begin{gathered}
\delta_{s, t}(B, \rho)=\left|\operatorname{Tr}\left\{\rho\left(\mathscr{L}_{t}(B)-\mathscr{L}_{s}(B)\right)\right\}\right| \rightarrow 0 \quad \text { при }|s-t| \rightarrow 0, \\
\varlimsup_{s \rightarrow t} \sup _{\|B\| \leqslant 1} \delta_{s, t}(B, \rho) \leqslant K_{t} \operatorname{Tr}(\rho \Lambda), \quad K_{t} \in L_{1}^{\operatorname{loc}}\left(\mathbb{R}_{+}\right) \quad \forall \rho \in \mathscr{T}_{\Lambda}(\mathscr{H}) .
\end{gathered}
$$

Строгое доказательство этих утверждений распадается на ряд вспомогательных лемм.

2. Априорные оценки и достаточные условия консервативности. Функциональная часть теории. В дальнейшем изложении нижний индекс '*' используется для обозначения квадратичных и билинейных форм, аргументы которых указьваются в квадратных скобках. Например, $\Lambda_{*}[\varphi, \psi]=\left(\Lambda^{1 / 2} \varphi, \Lambda^{1 / 2} \psi\right)$ - билинейная форма, порождаемая положительньм самосопряженньм оператором $\Lambda, \operatorname{dom} \Lambda_{*}=\operatorname{dom} \Lambda^{1 / 2}$ (см. [9]), и $\Lambda_{*}[\varphi]-$ соответствующая квадратичная форма. Неравенство $\Phi(I) \leqslant \Lambda$ означает, что $\operatorname{dom} \Lambda \subseteq \operatorname{dom} \Phi(I)$ и $(\psi, \Phi(I) \psi) \leqslant(\psi, \Lambda \psi) \forall \psi \in \operatorname{dom} \Lambda$. По непрерьвности это неравенство распространяется на $\psi \in \operatorname{dom} \Lambda^{1 / 2} \subseteq \operatorname{dom} \Phi(I)^{1 / 2}$.

В дальнейшем изложении предполагается, что для некоторого $N \geqslant 2$

$$
\mathscr{D}=\operatorname{dom} G^{N} \subseteq \operatorname{dom} \Lambda, \quad H \psi=H^{*} \psi \quad \forall \psi \in \operatorname{dom} G^{N} .
$$

Очевидно, что $\operatorname{dom} G^{N} \subseteq \operatorname{dom} H \cap \operatorname{dom} \Phi(I) \subseteq \operatorname{dom} G$, и если $H-$ самосопряженньй оператор, то, как было показано в [5], $\operatorname{dom} G \subseteq \operatorname{dom} \Phi_{*}(I)$. 
ЛЕмма 2.1. Если выполнены предположения (2.1) и

$$
-2 \operatorname{Re} \Lambda_{*}[G \varphi, \varphi] \leqslant c \Lambda_{*}[\varphi] \quad \forall \varphi \in \mathscr{D},
$$

mo

$$
A_{\lambda}(\Lambda)=\int_{0}^{\infty} d t e^{-\lambda t} W_{t}^{*} \Lambda W_{t} \leqslant(\lambda-c)^{-1} \Lambda
$$

для любого $\lambda>c u A_{\lambda}\left(\Lambda_{\varepsilon}\right) \leqslant(\lambda-c)^{-1} \Lambda_{\varepsilon}$ для любого $\varepsilon>0$, где $\Lambda_{\varepsilon}=\Lambda(I+\varepsilon \Lambda)^{-1}$.

ДокАЗАтЕЛЬСтво. Обозначим через $\mathscr{H}_{\Lambda}$ - гильбертово подпространство в $H$ со скалярньм произведением $(\psi, \varphi)=\left(\Lambda^{1 / 2} \psi, \Lambda^{1 / 2} \varphi\right)$. Если вьполнены условия леммы 2.1 , то полугруппа $W_{t}=\exp \{-G t\}$ непрерывна и ограничена в $\mathscr{H}_{\Lambda}$.

Действительно, для любого $\varphi \in \mathscr{D}$ имеем $\varphi_{t}=W_{t} \varphi \in \mathscr{D}$ и

$$
\frac{d}{d t}\left\|\varphi_{t}\right\|_{\Lambda}^{2}=-2 \operatorname{Re} \Lambda_{*}\left[G \varphi_{t}, \varphi_{t}\right] \leqslant c\left\|\varphi_{t}\right\|_{\Lambda}^{2},
$$

т.е. $\left\|W_{t} \varphi\right\|_{\Lambda}^{2} \in C^{1}\left(\mathbb{R}_{1}\right)$ и $\left\|W_{t} \varphi\right\|_{\Lambda}^{2} \leqslant e^{c t}\|\varphi\|_{\Lambda}^{2}$. Оба утверждения распространяются по непрерьвности на $\operatorname{dom} \Lambda_{*}$. Это свойство полугрупшы $W_{t}$ используется в [4] для корректного определения интегральной формы уравнения Линдблада. Здесь мы воспользуемся тем, что $\left\|\varphi_{t}\right\|_{\Lambda}^{2}=\Lambda_{*}\left[\varphi_{t}\right]-$ непрерывная функция параметра $t \in \mathbb{R}_{+}$.

Рассмотрим плотно определенную квадратичную форму $A_{\lambda}(\Lambda)_{*}$, соответствующую оператору $A_{\lambda}(\Lambda)$ :

$$
\begin{aligned}
\lambda A_{\lambda}(\Lambda)_{*}[\varphi] & =-\int_{0}^{\infty} \Lambda_{*}\left[\varphi_{t}\right] d e^{-\lambda t}=\Lambda_{*}[\varphi]-2 \operatorname{Re} \int_{0}^{\infty} d t e^{-\lambda t} \Lambda_{*}\left[G \varphi_{t}, \varphi_{t}\right] \\
& \leqslant \Lambda_{*}[\varphi]+c \int_{0}^{\infty} d t e^{-\lambda t} \Lambda_{*}\left[\varphi_{t}\right]=\Lambda_{*}[\varphi]+c A_{\lambda}(\Lambda)_{*}[\varphi] .
\end{aligned}
$$

Таким образом, $A_{\lambda}(\Lambda)_{*}[\varphi] \leqslant(\lambda-c)^{-1} \Lambda_{*}[\varphi]$ для всех $\varphi \in \mathscr{D}$, т.е. $A_{\lambda}(\Lambda) \leqslant(\lambda-c)^{-1} \Lambda$ на $\operatorname{dom} \Lambda$.

В работе [6] было показано, что для любого сжимающего вполне положительного отображения $\pi(\cdot)$, любого положительного самосопряженного оператора $B$ и любого $\varepsilon \in(0, \mu)$ имеют место оценки

$$
\begin{aligned}
\pi\left(B_{\varepsilon}\right) \leqslant \pi\left(B_{\varepsilon-\mu}\right)_{\mu} \leqslant \pi(B)_{\varepsilon} \quad \forall B \in \mathscr{B}_{+}(\mathscr{H}), \quad B_{\varepsilon}=B(I+\varepsilon B)^{-1} \\
0 \leqslant X_{\varepsilon} \leqslant Y_{\varepsilon}
\end{aligned}
$$

если $0 \leqslant X \leqslant Y$ - самосопряженные операторы. Полагая в $(2.2) \pi(\cdot)=A_{\lambda}(\cdot), B=\Lambda$, получаем последнее утверждение леммы. Лемма доказана.

Пусть для некоторого $c \in \mathbb{R}_{+}$

$$
\mathscr{L}(\Lambda)_{*}[\varphi] \leqslant c \Lambda_{*}[\varphi] \quad \forall \varphi \in \mathscr{D},
$$

где $\mathscr{L}(B)_{*}[\varphi]=\Phi(B) *[\varphi]-(G \varphi, B \varphi)-(B \varphi, G \varphi)$. 
ТЕОРема 2.1. Если выполнены условия (2.1), (2.4), то

$$
R_{\lambda}^{\min }\left(\Lambda_{\varepsilon}\right) \leqslant(\lambda-c)^{-1} \Lambda_{\mu}, \quad P_{t}^{\min }\left(\Lambda_{\varepsilon}\right) \leqslant e^{c t} \Lambda_{\mu}
$$

для любого $\lambda>c, \mu \in(0, \varepsilon)$, где $\Lambda_{\varepsilon}=\Lambda(I+\varepsilon \Lambda)^{-1} \in \mathscr{B}_{+}(\mathscr{H})$.

ДокАЗАТЕЛЬСТво. Докажем, что

$$
R_{\lambda}^{\min }\left(\Lambda_{\varepsilon}\right)=\sup _{n} R_{\lambda}^{(n)}\left(\Lambda_{\varepsilon}\right) \leqslant(\lambda-c)^{-1} \Lambda_{\mu} \quad \forall \mu \in(0, \varepsilon),
$$

где уравнение для минимальной резольвенты следует из рекуррентных уравнений для минимальной динамической полугруппы (см. [4], [5])

$$
R_{\lambda}^{(n)}\left(\Lambda_{\varepsilon}\right)=A_{\lambda}\left(\Lambda_{\varepsilon}\right)+Q_{\lambda}\left(R^{(n-1)}\left(\Lambda_{\varepsilon}\right)\right), \quad R_{\lambda}^{(0)}=0 .
$$

Поскольку условие леммы 2.1 следует из (2.4), то неравенство

$$
R_{\lambda}^{(k)}\left(\Lambda_{\varepsilon}\right)=A_{\lambda}\left(\Lambda_{\varepsilon}\right) \leqslant(\lambda-c)^{-1} \Lambda_{\mu} \quad \forall \mu \in(0, \varepsilon)
$$

при $k=1$ является следствием леммы 2.1 и оценки $(2.2)$ для $\pi(\cdot)=A_{\lambda}(\cdot), B=\Lambda$. Предположим, что это неравенство вьполнено для $k=2,3, \ldots, n-1$, и докажем его для $k=n$.

Из свойств $(2.2),(2.3)$ регуляризующего отображения $A \rightarrow A_{\varepsilon}$ относительно сжимающего вполне положительного отображения $R_{\lambda}^{(n)}(\cdot)$ мы получаем

$$
R_{\lambda}^{(n)}\left(\Lambda_{\varepsilon}\right) \leqslant\left(R_{\lambda}^{(n)}\left(\Lambda_{\varepsilon-\mu}\right)\right)_{\mu} \quad \forall \mu \in(0, \varepsilon),
$$

а из предположения индукции $R_{\lambda}^{(n-1)}\left(\Lambda_{\varepsilon}\right) \leqslant(\lambda-c)^{-1} \Lambda_{\varepsilon-\mu}$ следует, что

$$
R_{\lambda}^{(n)}\left(\Lambda_{\varepsilon}\right) \leqslant\left\{A_{\lambda}\left(\Lambda_{\varepsilon-\mu}\right)+\frac{1}{\lambda-c} \int_{0}^{\infty} d t e^{-\lambda t} W_{t}^{*} \Phi\left(\Lambda_{\varepsilon-\mu}\right) W_{t}\right\}_{\mu} .
$$

Оценка (2.4) обеспечивает неравенство для квадратичных форм на $\mathscr{D}$ :

$$
\Phi\left(\Lambda_{\varepsilon-\mu}\right)_{*} \leqslant \sup _{\varepsilon>0} \Phi\left(\Lambda_{\varepsilon}\right)_{*} \leqslant\left(c \Lambda+G^{*} \Lambda+\Lambda G\right)_{*} .
$$

Поэтому для любого $\varphi \in \mathscr{D}$ выполнено

$$
\begin{aligned}
R_{\lambda}^{(n)}\left(\Lambda_{\varepsilon}\right)_{*}[\varphi] & \leqslant\left(\left\{A_{\lambda}\left(\Lambda_{\varepsilon-\mu}\right)+\frac{1}{\lambda-c} \int_{0}^{\infty} d t e^{-\lambda t} W_{t}^{*} \Phi\left(\Lambda_{\varepsilon-\mu}\right) W_{t}\right\}_{\mu}\right)_{*}[\varphi] \\
& \leqslant\left(\left\{A_{\lambda}\left(\Lambda_{\varepsilon-\mu}\right)+\frac{c}{\lambda-c} A_{\lambda}(\Lambda)-\frac{1}{\lambda-c} \int_{0}^{\infty} d t e^{-\lambda t} \frac{d}{d t}\left(W_{t}^{*} \Lambda_{*} W_{t}\right)\right\}_{\mu}\right)_{*}[\varphi] \\
& \leqslant\left(\left\{A_{\lambda}(\Lambda)\left(1+\frac{c}{\lambda-c}\right)+\frac{1}{\lambda-c} \Lambda-\frac{\lambda}{\lambda-c} A_{\lambda}(\Lambda)\right\}_{\mu}\right)_{*}[\varphi] \\
& =(\lambda-c)^{-1}\left(\Lambda_{\mu}\right)_{*}[\varphi] .
\end{aligned}
$$


Таким образом, $R_{\lambda}^{(n)}\left(\Lambda_{\varepsilon}\right)_{*}[\varphi] \leqslant(\lambda-c)^{-1}\left(\Lambda_{\mu}\right)_{*}[\varphi]$ для всех $\mu \in(0, \varepsilon), n=1,2, \ldots$ и всех $\varphi \in \mathscr{D}$. Поскольку $\Lambda_{\varepsilon}, \Lambda_{\mu}$ - ограниченные операторы, то отсюда мы заключаем, что $R_{\lambda}^{\min }\left(\Lambda_{\varepsilon}\right) \leqslant(\lambda-c)^{-1} \Lambda_{\mu}$ для любых $1 \geqslant \varepsilon>\mu>0$. В частности, при $\lambda=(t / n)^{-1}$ имеем $\lambda R_{\lambda}^{\min }\left(\Lambda_{\varepsilon}\right) \leqslant(1-c t / n)^{-1} \Lambda_{\mu}$. Для завершения доказательства воспользуемся стандартным представлением сжимающей полугруппы через ее резольвенту (см. [9]):

$$
P_{t}^{\min }\left(\Lambda_{\varepsilon}\right)=s-\lim _{n \rightarrow \infty}\left(\lambda R_{\lambda}^{\min } \cdots\left(\lambda R_{\lambda}^{\min }\left(\Lambda_{\mu}\right)\right)\right) \leqslant \Lambda_{\mu} \lim _{n \rightarrow \infty}\left(1-\frac{c t}{n}\right)^{-n}=e^{c t} \Lambda_{\mu} .
$$

Теорема доказана.

Из этой теоремы вытекает оценка резольвенты минимальной динамической полугруппы и самой полугруппы, если отображение $\Phi(\cdot)$ обладает свойством обобщенной нормальности.

ОПРЕДЕЛЕНИЕ 2.1. Вполне положительное отображение $\Phi(\cdot)$ удовлетворяет обобщенному условию нормальности относительно оператора $\Lambda$, если

$$
\sup _{\varepsilon \in(0,1]} \Phi\left(T_{\varepsilon}\right)_{*}[\varphi]=\Phi(T)_{*}[\varphi] \quad \forall \varphi \in \mathscr{D}, \quad T_{\varepsilon}=T(I+\varepsilon T)^{-1} \in \mathscr{B}_{+}(\mathscr{H})
$$

для любого положительного самосопряженного оператора $T$ такого, что $T \leqslant c_{T} \Lambda$ с некоторой постоянной $c_{T} \in \mathbb{R}_{+}$.

Как мы увидим ниже, предположение (2.6) означает, что преддвойственное отображение $\Phi^{\dagger}(\cdot)$ сопоставляет оператору $\rho_{\varphi}=|\varphi\rangle\langle\varphi|$ ранга 1 оператор $\Phi^{\dagger}\left(\rho_{\varphi}\right)$ с конечным следом такой, что для любого положительного оператора $T \leqslant c_{T} \Lambda$ плотно определенный оператор $\Phi^{\dagger}\left(\rho_{\varphi}\right) T$ допускает ограниченное продолжение на $\mathscr{H}$ и имеет конечньй след

$$
\operatorname{Tr}\left\{\Phi^{\dagger}\left(\rho_{\varphi}\right) T\right\}=\sup _{\varepsilon \in(0,1]} \operatorname{Tr}\left\{\Phi^{\dagger}\left(\rho_{\varphi}\right) T_{\varepsilon}\right\}=\Phi(T)_{*}[\varphi]
$$

В этих обозначениях условие обобщенной нормальности означает, что $\Phi^{\dagger}(\cdot): L_{\mathscr{D}} \rightarrow \mathscr{T}_{\Lambda}$, где $L_{\mathscr{D}}$ - линейная оболочка, порождаемая проекторами $\rho_{\varphi}, \varphi \in \mathscr{D}$.

ТЕОрема 2.2. Пусть $T$ - положительный замкнутый оператор такой, что $T \leqslant c_{T} \Lambda$. Тогда при условиях (2.1), (2.4), (2.6) существует наименьшая верхняя грань

$$
P_{t}^{\min }(T)=\text { l.u.b. } \varepsilon P_{t}^{\min }\left(T_{\varepsilon}\right)=P_{t}^{\min }\left(\text { l.u.b. } \varepsilon T_{\varepsilon}\right) \leqslant c_{T} e^{c t} \Lambda, \quad \varepsilon \in(0,1] \text {. }
$$

ДокАЗАТЕльство. Рассмотрим последовательность $P_{t}^{(n)}\left(T_{\varepsilon}\right)$, сильно сходящуюся при $n \rightarrow \infty$ к $P_{t}^{\min }\left(T_{\varepsilon}\right)$. Из теоремы 2.1 имеем

$$
P_{t}^{(n)}\left(T_{\varepsilon}\right) \leqslant P_{t}^{\min }\left(T_{\varepsilon}\right) \leqslant c_{T} P_{t}^{\min }\left(\Lambda_{\varepsilon}\right) \leqslant c_{T} e^{c t} \Lambda_{\mu}
$$

для любого $\mu \leqslant \varepsilon$. Покажем по индукции, что

$$
\text { l.u.b. } P_{t}^{(n)}\left(T_{\varepsilon}\right)=P_{t}^{(n)}\left(\text { l.u.b. } T_{\varepsilon}\right) \text {, }
$$


где $T_{\varepsilon}=T\left(I+T_{\varepsilon}\right)^{-1} \in \mathscr{B}_{+}(\mathscr{H})$. Рассмотрим три объекта:

(1) вполне положительное отображение $P_{t}^{(1)}\left(T_{\varepsilon}\right)=W_{t}^{*} T_{\varepsilon} W_{t}$;

(2) последовательность векторов из $\mathscr{H}$

$$
\varphi_{\varepsilon}=(I+\varepsilon T)^{-1} W_{t} \varphi \in \operatorname{dom} \Lambda \subseteq \operatorname{dom} T \quad \forall \varphi \in \mathscr{D},
$$

сильно сходящуюся к $W_{t} \varphi$ при $\varepsilon \rightarrow 0$;

(3) возрастающую последовательность квадратичных форм

$$
\left(T_{\varepsilon}\right)_{*}\left[W_{t} \varphi\right]=(T)_{*}\left[W_{t} \varphi_{\varepsilon}\right] \leqslant(T)_{*}\left[W_{t} \varphi_{\mu}\right] \leqslant c_{T} \Lambda_{*}\left[W_{t} \varphi\right], \quad 0<\mu<\varepsilon .
$$

Поскольку $T$ - замкнутьй оператор, то

$$
\sup _{\varepsilon}\left(T_{\varepsilon}\right)_{*}\left[\varphi_{t}\right]=\sup _{\varepsilon} P_{t}^{(1)}\left(T_{\varepsilon}\right)_{*}[\varphi]=T_{*}\left[\varphi_{t}\right]=P_{t}^{(1)}(T)_{*}[\varphi], \quad \varepsilon \in(0,1] .
$$

То есть l.u.b. $P_{t}^{(1)}\left(T_{\varepsilon}\right)=P_{t}^{(1)}(T)$ на $\mathscr{D}$. Предположим, что равенство

$$
\sup _{\varepsilon}\left(P_{t}^{(k)}\left(T_{\varepsilon}\right)\right)_{*}=\left(P_{t}^{(k)}(T)\right)_{*}
$$

для соответствуюших квадратичных форм на $\mathscr{D}$ верно при $k=2,3, \ldots, n-1$, и докажем его при $k=n$.

Рассмотрим рекуррентные уравнения для $P_{t}^{(n)}$ :

$$
P_{t}^{(n)}\left(T_{\varepsilon}\right)_{*}[\varphi]=P_{t}^{(1)}\left(T_{\varepsilon}\right)_{*}[\varphi]+\int_{0}^{t} d s \Phi_{*}\left(P_{s}^{(n-1)}\left(T_{\varepsilon}\right)\right)\left[W_{t-s} \varphi\right],
$$

где $P_{s}^{(n)}\left(T_{\varepsilon}\right) \leqslant c_{T} P_{s}^{(n)}\left(\Lambda_{\varepsilon}\right) \leqslant c_{T} e^{c t} \Lambda_{\mu}$ для любого $\mu \in(0, \varepsilon)$. Тогда из условия обобщенной нормальности (2.6) и предположения индукции вытекает абсолютная интегрируемость верхней границы относительно $s \in[0, t]$ :

$$
\sup _{\varepsilon} \Phi_{*}\left(P_{s}^{(n)}\left(T_{\varepsilon}\right)\right)\left[W_{t-s} \varphi\right]=\Phi_{*}\left(P_{s}^{(n)}\left(\sup _{\varepsilon} T_{\varepsilon}\right)\right)\left[W_{t-s} \varphi\right] \in \mathscr{L}_{1}^{\text {loc }}\left(\mathbb{R}_{+}\right) .
$$

Следовательно, теорема Лебега о предельном переходе под знаком интеграла может быть применена к рекуррентной последовательности (2.7). Отсюда вытекает переста-

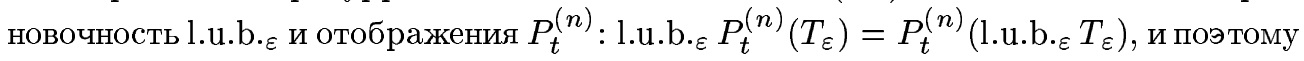

$$
P_{t}^{\min }(T)=\text { l.u.b.n l.u.b. } \varepsilon P_{t}^{(n)}\left(T_{\varepsilon}\right) \geqslant \text { l.u.b. l.u.b. }{ }_{n} P_{t}^{(n)}\left(T_{\varepsilon}\right)=\widetilde{P}_{t}(T) .
$$

С другой стороны, из интегрального уравнения для $\widetilde{P}_{t}(\cdot)$ имеем

$$
\begin{aligned}
\widetilde{P}_{t}(T)_{*}[\varphi] & =\sup _{\varepsilon} P_{t}\left(T_{\varepsilon}\right)_{*}[\varphi]=\sup _{\varepsilon}\left\{P_{t}^{(1)}\left(T_{\varepsilon}\right)_{*}[\varphi]+\int_{0}^{t} d s \Phi_{*}\left(\widetilde{P}_{s}\left(T_{\varepsilon}\right)\right)\left[W_{t-s} \varphi\right]\right\} \\
& \leqslant P_{t}^{(1)}(T)_{*}[\varphi]+\int_{0}^{t} d s \Phi_{*}\left(\widetilde{P}_{s}(T)\right)\left[W_{t-s} \varphi\right] .
\end{aligned}
$$

Отсюда мы заключаем, что $\widetilde{P}_{t}(T) \leqslant P_{t}^{\min }(T)$. Вместе с (2.8) это дает утверждение теоремы. 
СЛЕДСТВИЕ 2.1. Если выполнены условия (2.1), (2.6), то неравенство (2.4) является достаточным для консервативности минимальной динамической полугруппь.

Это утверждение вытекает из равномерной ограниченности убывающей последовательности ограниченных операторов $Q_{\lambda}^{(n)}(I)$ и слабой сходимости ряда

$$
\sum_{1}^{\infty} Q_{\lambda}^{k}(I) \leqslant R_{\lambda}^{\min }(\Lambda) \leqslant(\lambda-c)^{-1} \Lambda
$$

на плотном в $\mathscr{H}$ множестве $\mathscr{D}$.

Теорема 2.3. Если выполнены условия (2.1), (2.4), (2.6), то для любого замыкаемого положительного оператора $T \leqslant c_{T} \Lambda$ функиия $P_{t}^{\min }(T)_{*}[\varphi], \varphi \in \mathscr{D}$, непрерывна по параметру $t \in \mathbb{R}_{+}$.

ДоКАЗАТЕЛЬСтво. Воспользуемся интегральным уравнением для квадратичной формы $P_{t}^{\min }(T)_{*}[\varphi]($ см. $[4]-[6])$ :

$$
P_{t}^{\min }(T)_{*}[\varphi]=T_{*}\left[\varphi_{t}\right]+\int_{0}^{t} \Phi_{*}\left(P_{s}^{\min }(T)\right)\left[\varphi_{t-s}\right] d s
$$

Поскольку $T \leqslant c_{T} \Lambda$, то $\operatorname{dom} T^{1 / 2} \supseteq \operatorname{dom} \Lambda^{1 / 2}$. Следовательно, функция

$$
v_{t}=\Lambda^{-1 / 2} T^{1 / 2} W_{t} \varphi, \quad \varphi \in \mathscr{D},
$$

принадлежит $\operatorname{dom} \Lambda_{*}$ и сходится к $\varphi$ при $t \rightarrow 0$. Теперь из замькаемости квадратичной формы $T_{*}\left[\varphi_{t}\right]=\left\|T^{1 / 2} W_{t} \varphi\right\|^{2}=\left\|\Lambda^{1 / 2} v_{t}\right\|^{2}$ следует непрерывность первого слагаемого в $(2.9)$.

Нетрудно видеть, что для любого $\varepsilon>0$ функция параметра $s \in(0, t)$

$$
\Phi_{s}^{(\varepsilon)}=\Phi_{*}\left(P_{s}^{\min }\left(T_{\varepsilon}\right)\right)\left[\varphi_{t-s}\right]
$$

непрерьвна для любого $\varphi \in \mathscr{D}$. Поэтому ее верхняя грань

$$
\Phi_{s}=\sup _{\varepsilon>0} \Phi_{*}\left(P_{s}^{\min }\left(T_{\varepsilon}\right)\right)\left[\varphi_{t-s}\right] \geqslant 0
$$

является измеримой функцией. Покажем, что $\Phi_{s} \in \mathscr{L}_{1}^{\operatorname{loc}}\left(\mathbb{R}_{+}\right)$. Из условия $(2.4)$ имеем

$$
\begin{aligned}
0 & \leqslant \Phi_{s}=\Phi_{*}\left(P_{s}^{\min }(T)\right)\left[\varphi_{t-s}\right]=\mathscr{L}_{*}(\Lambda)\left[\varphi_{t-s}\right]+2 \operatorname{Re} \Lambda_{*}\left[\varphi_{t-s}, G \varphi_{t-s}\right] \\
& \leqslant c \Lambda_{*}\left[\varphi_{t-s}\right]+2 \operatorname{Re} \Lambda_{*}\left[\varphi_{t-s}, G \varphi_{t-s}\right]<\infty
\end{aligned}
$$

так как $G \varphi_{t-s}=W_{t-s} G \varphi \in \operatorname{dom} \Lambda_{*}$ и оба слагаемьх непрерывны по $t$. Следовательно, они локально ограничены, и это дает абсолютную непрерывность второго слагаемого в (2.9). Теорема доказана. 
3. Априорные оценки для преддвойственной полугруппы. В этом разделе используется двойственность между алгебрами $\mathscr{B}(\mathscr{H})$ и $\mathscr{T}(\mathscr{H})$, устанавливаемая отделяющей билинейной формой $\operatorname{Tr} \rho B, \rho \in \mathscr{T}(\mathscr{H}), B \in \mathscr{B}(\mathscr{H})$.

Обозначим через $T_{t}(\cdot): \mathscr{T}(\mathscr{H}) \rightarrow \mathscr{T}(\mathscr{H})$ динамическую полугруппу в шрёдингеровском представлении преддвойственную полугруппе $P_{t}(\cdot): \operatorname{Tr}\left\{T_{t}(\rho) B\right\}=\operatorname{Tr}\left\{\rho P_{t}(B)\right\}$. Пусть $\mathscr{H}$ - сепарабельное гильбертово пространство и $\Lambda \geqslant \Phi(I) \geqslant I$. В теории динамических полугрупп эти предположения не являются ограничительными (см. [4]).

ОПРЕДЕЛЕНИЕ 3.1. Пусть $\Lambda$ - положительньй самосопряженный оператор. Обозначим через $\mathscr{T}_{\Lambda}(\mathscr{H}) \subseteq \mathscr{T}(\mathscr{H})$ множество операторов из линейной оболочки конуса $\mathscr{T}_{\Lambda}^{+}(\mathscr{H})$ положительных операторов $\rho$ таких, что

(1) плотно определенньй оператор $\rho \Lambda$ может быть продолжен до ограниченного оператора на $\mathscr{H}$;

(2) для любой полной ортонормированной системы $\left\{\psi_{k}\right\}$ ряд

$$
\sum\left|\left(\psi_{k}, \rho \Lambda \psi_{k}\right)\right|
$$

абсолютно сходится.

В этом случае следом оператора $\rho \Lambda$ назьвается

$$
\operatorname{Tr}(\rho \Lambda)=\sum_{k}\left(\psi_{k}, \rho \Lambda \psi_{k}\right)
$$

Нетрудно видеть, что с одной стороны

$$
\lim _{\varepsilon \rightarrow+0}\left(\psi, \rho \Lambda_{\varepsilon} \psi\right)=(\psi, \rho \Lambda \psi)
$$

для любого $\psi \in \operatorname{dom} \Lambda$, причем обе части этого равенства однозначно продолжаются на все пространство $\mathscr{H}$. С другой стороны, $\left(\psi, \rho \Lambda_{\varepsilon} \psi\right)=\left\|\rho^{1 / 2} \Lambda_{\varepsilon}^{1 / 2} \psi\right\|^{2} \geqslant 0$, где $\Lambda_{\varepsilon}=\Lambda(I+\varepsilon \Lambda)^{-1}$. Поэтому имеет место следующая теорема.

TEOрема 3.1. Если $\rho \in \mathscr{T}_{\Lambda}^{+}(\mathscr{H})$, mo

(1) для любой полной ортонормированной системы $\left\{\psi_{k}\right\}$

$$
\operatorname{Tr} \rho \Lambda=\sup _{n} \sum_{k=1}^{n}\left(\psi_{k}, \rho \Lambda \psi_{k}\right)=\sum_{k=1}^{\infty} \sup _{\varepsilon>0}\left(\psi_{k}, \rho \Lambda_{\varepsilon} \psi_{k}\right)=\sup _{\varepsilon>0} \operatorname{Tr}\left(\rho \Lambda_{\varepsilon}\right) \geqslant 0
$$

(2) если выполнены условия теоремы 2.2, то преддвойственная полугруппа $T_{t}$ действует в $\mathscr{T}_{\Lambda}(\mathscr{H})$ :

$$
0 \leqslant \operatorname{Tr}\left\{T_{t}(\rho) \Lambda\right\} \leqslant e^{c t} \operatorname{Tr} \rho \Lambda
$$

(3) если $A$ - положительный самосопряженный оператор такой, что $0 \leqslant$ $A_{*}[\varphi] \leqslant \Lambda_{*}[\varphi]$, mо $\operatorname{Tr}(\rho A) \leqslant \operatorname{Tr}(\rho \Lambda)$ для любого $\rho \in \mathscr{T}_{\Lambda}^{+}(\mathscr{H})$. 
ДокАЗАТЕльство. (1) Первая часть леммы вытекает из свойств следа и возможности почленного предельного перехода при $\varepsilon \rightarrow+0$ в сумме $\sum\left(\psi_{k}, \rho \Lambda_{\varepsilon} \psi_{k}\right)$, поскольку ряд равномерно ограничен и абсолютно сходится. Более точно, для каждого $n$ остаток

$$
\left|\sum_{k=n}^{\infty}\left(\psi_{k}, \rho \Lambda_{\varepsilon} \psi_{k}\right)\right| \leqslant \sum_{k=n}^{\infty}\left|\left(\psi_{k}, \rho \Lambda \psi_{k}\right)\right|
$$

имеет равномерную по $\varepsilon$ оценку и сходится к нулю при $n \rightarrow \infty$, а $\varepsilon(n)$ можно выбрать так, что при всех $\varepsilon \in(0, \varepsilon(n))$

$$
\sum_{k=1}^{n}\left|\left(\psi_{k}, \rho\left(\Lambda-\Lambda_{\varepsilon}\right) \psi_{k}\right)\right| \leqslant n^{-1} .
$$

Заметим, что если $A, B$ - положительные самосопряженные операторы, $A \leqslant B$, и оператор $B$ обратим на образе $A$, то существование ограниченного продолжения $\rho A=\rho B B^{-1} A$ следует из ограниченности оператора $B^{-1} A$ и существования ограниченного продолжения оператора $\rho B$. Поэтому утверждение $(2)$ вытекает из теоремы 2.2 и определения полугрупшы $T_{t}(\cdot)$ :

$$
\operatorname{Tr}\left\{T_{t}(\rho) \Lambda\right\}=\operatorname{Tr}\left\{\rho P_{t}(\Lambda)\right\} \leqslant e^{c t} \operatorname{Tr}(\rho \Lambda) \quad \forall \rho \in \mathscr{T}_{\Lambda}^{+}(\mathscr{H}) .
$$

(3) Последняя часть теоремы доказывается от противного. Предположим, что $\operatorname{Tr}(\rho A) \geqslant \operatorname{Tr}(\rho \Lambda)+\delta$. Тогда из доказанной вьше первой части теоремы следует, что существует $\varepsilon=\varepsilon(\delta)$ такое, что $\operatorname{Tr}\left(\rho A_{\varepsilon}\right) \geqslant \operatorname{Tr}\left(\rho \Lambda_{\varepsilon}\right)+\delta / 2$, т.е.

$$
\sum_{k}\left(\psi_{\rho, k}, A_{\varepsilon} \psi_{\rho, k}\right) \geqslant \sum_{k}\left(\psi_{\rho, k}, \Lambda_{\varepsilon} \psi_{\rho, k}\right)+\frac{\delta}{2}
$$

где $\psi_{\rho, k}=\rho^{1 / 2} \psi_{k} \in \operatorname{dom} \Lambda^{1 / 2} \subseteq \operatorname{dom} A^{1 / 2}$, a $\left\{\psi_{k}\right\}$ - полная ортонормированная системa.

Теперь неравенство (3.1) противоречит наложенному вьше условию (3):

$$
\sup _{\varepsilon}\left(\psi_{\rho, k}, A_{\varepsilon} \psi_{\rho, k}\right)=A_{*}\left[\psi_{\rho, k}\right] \leqslant \Lambda_{*}\left[\psi_{\rho, k}\right]=\sup _{\varepsilon}\left(\psi_{\rho, k}, \Lambda_{\varepsilon} \psi_{\rho, k}\right) .
$$

Теорема доказана.

СледСТВИЕ 3.1. При условиях теоремы 2.3 функиия $R_{t}=\operatorname{Tr}\left\{\rho P_{t}^{\min }(T)\right\}$ непрерывна по параметру $t \in \mathbb{R}_{+}$для любого $\rho \in \mathscr{T}_{\Lambda}(\mathscr{H})$.

Действительно, множество чистых состояний $\rho_{\varphi}, \varphi \in \operatorname{dom} \Lambda^{1 / 2}$, является множеством крайних точек алгебры $\mathscr{T}_{\Lambda}(\mathscr{H})=\mathscr{T}\left(\mathscr{H}_{\Lambda}\right)$ (см. [9, теорема 2.3.15]). Поэтому непрерьвность $R_{t}$ вытекает из непрерьвности $P_{t}^{\min }(T)_{*}[\varphi]=\operatorname{Tr}\left\{\rho_{\varphi} P_{t}^{\min }(T)\right\}$, доказанной выше в теореме 2.3 для чистых состояний $\rho_{\varphi}=|\varphi\rangle\langle\varphi|$.

В работе [4] был введен конус вполне положительных нормальных отображений $\operatorname{CPn}\left(\mathscr{H}_{\Lambda}\right)$, определяемый как пополнение множества вполне положительных отображений $\operatorname{CPn}(\mathscr{H})$, относительно локально выпуклой топологии, задаваемой системой полунорм

$$
\sigma_{A, B}(\Phi)=\sup _{X \in B, \psi \in A}\left|\Phi_{*}(B)[\psi]\right|,
$$

где $A$ и $B$ - абсолютно вьпуклые компактные подмножества из $\mathscr{H}_{\Lambda}$ и $\mathscr{B}(\mathscr{H})$ соответственно. Теперь мы можем дать еще одно эквивалентное описание конуса $\mathrm{CPn}\left(\mathscr{H}_{\Lambda}\right)$. 
Теорема 3.2. Конус $\mathrm{CPn}\left(\mathscr{H}_{\Lambda}\right)$ состоит из вполне положительных отображений $\Phi(\cdot)$, двойственных отображениям $\Phi^{\dagger}(\cdot): \mathscr{T}_{\Lambda}(\mathscr{H}) \rightarrow \mathscr{T}(\mathscr{H})$ :

$$
\Phi_{*}(B)[\psi]=\operatorname{Tr}\left\{\Phi^{\dagger}\left(\rho_{\psi}\right)\right\}, \quad \rho_{\psi}=|\psi\rangle\langle\psi| .
$$

ДокАЗАТЕЛЬСТво. Поскольку $\Phi_{*}(\cdot)[\psi]: \mathscr{B}(\mathscr{H}) \rightarrow \mathbb{C}$-нормальное состояние, задаваемое квадратичной формой $\Phi_{*}(B)[\psi]=\operatorname{Tr}\left(B \Phi^{\dagger}\left(\rho_{\psi}\right)\right)$, то из (3.2) и [9, теорема 2.4.21] следует, что $\Phi^{\dagger}\left(\rho_{\psi}\right) \in \mathscr{T}(\mathscr{H})$ для любого $\psi \in \mathscr{D}$. Теперь из части (3) теоремы 3.1 следует, что $\Phi^{\dagger}(\rho) \in \mathscr{T}(\mathscr{H})$ для любого $\rho \in \mathscr{T}_{\Lambda}(\mathscr{H})$, поскольку утверждения теоремы 3.1 сохраняют силу на пополнении линейной оболочки множества чистых состояний в $\mathscr{T}_{\Lambda}(\mathscr{H})=\mathscr{T}\left(\mathscr{H}_{\Lambda}\right)$.

Обратно, пусть $\Phi^{\dagger}(\cdot): \mathscr{T}_{\Lambda}(\mathscr{H}) \rightarrow \mathscr{T}(\mathscr{H})$ и пусть $\Phi_{*}^{(\varepsilon)}(B)[\psi]=\operatorname{Tr}\left\{B \sigma_{\varepsilon}\right\}$, где $\sigma_{\varepsilon}=\Phi^{\dagger}\left(\rho_{\psi}^{(\varepsilon)}\right), \rho_{\psi}^{(\varepsilon)}=\left|\psi_{\varepsilon}\right\rangle\left\langle\psi_{\varepsilon}\right|, \psi_{\varepsilon}=(I+\varepsilon \Lambda)^{-1} \psi$. Нетрудно видеть, что каждая квадратичная форма $\Phi_{*}^{(\varepsilon)}(B)[\psi]$ является нормальной и допускает ограниченное продолжение на $\mathscr{H}$, так как может быть записана в виде конечного следа ограниченного оператора: $\Phi_{*}^{(\varepsilon)}(B)[\psi]=\operatorname{Tr}\left(B \sigma_{\varepsilon}\right)$, где $\sigma_{\varepsilon}-$ положительный оператор с конечным следом

$$
\operatorname{Tr} \sigma_{\varepsilon}=\Phi_{*}^{(\varepsilon)}(I)[\psi] \leqslant \Lambda\left[\psi_{\varepsilon}\right] \leqslant \varepsilon^{-2}\|\psi\|^{2} .
$$

Нетрудно видеть, что $\Phi_{*}^{(\varepsilon)}(B)[\psi] \rightarrow \Phi_{*}(B)[\psi]$ при $\varepsilon \rightarrow 0$ для любого $\psi \in \mathscr{H}_{\Lambda}$ и эта сходимость равномерна на компактных множествах в $\mathscr{H}_{\Lambda}$ и $\mathscr{B}(\mathscr{H})$.

4. Пополнение множества регулярных инфинитезимальных отображений с кусочно постоянными коэффициентами. Рассмотрим уравнение марковской эволюции

$$
\frac{d}{d t} P_{s, t}(B)=\mathscr{L}_{t}\left(P_{s, t}(B)\right),\left.\quad P\right|_{t=s}(B)=B, \quad 0 \leqslant s \leqslant t,
$$

с инфинитезимальным отображением $\mathscr{L}_{t}(\cdot)$, являющимся простой функцией времени, т.е. принимающей постоянные значения на интервалах, число которых конечно на любом конечном подмножестве $\mathbb{R}_{+}$:

$$
\mathscr{L}_{t}(\cdot)=\mathscr{L}_{i}(\cdot), \quad \Phi_{t}(\cdot)=\Phi_{i}(\cdot), \quad H_{t}=H_{i} \quad \forall t \in\left(t_{i-1}, t_{i}\right], \quad t_{0}=0, \quad i>0 .
$$

Решение $P_{t, s}(\cdot)$ уравнения (4.1), построенное как композиция решений уравнений с постоянньми коэффициентами, обладает характеристическим свойством (левого) коцикла: $P_{t, \tau} P_{\tau, s}=P_{t, s}, s<\tau<t$, и назьвается марковским коииклом:

$$
P_{t, s}(\cdot)=P_{t-t_{n}}^{(n)}\left(\cdots P_{t_{k(s)}-s}^{(k(s))}(\cdot)\right)
$$

где $P_{t}^{(k)}(\cdot)=e^{t \mathscr{L}_{k}}(\cdot)$ - минимальная динамическая полугруппа с генератором $\mathscr{L}_{k}(\cdot)$, постоянньм на полуинтервале $\left(t_{k-1}, t_{k}\right], k(s)=\left\{\min k: t_{k}>s\right\}, t_{n}=\left\{\max t_{j}: t_{j}<t\right\}$.

Ясно, что если существует последовательность вполне положительных консервативных марковских коциклов, сходящаяся ультраслабо или слабо при каждом $t \in \mathbb{R}_{+}$, то предел является вполне положительным консервативным марковским коциклом. Рассмотрим сначала априорные оценки для решений неоднородных уравнений Линдблада с простыми коэффищиентами.

Пусть $D_{t}-$ семейство замкнутых операторов таких, что

$$
\left(D_{t}\right)_{*}[\varphi] \leqslant c_{D}(t) \Lambda_{*}[\varphi], \quad \mathscr{L}_{t}(\Lambda)_{*}[\varphi] \leqslant c_{\Lambda}(t) \Lambda_{*}[\varphi]
$$

$c_{D}(t), c_{\Lambda}(t) \in L_{1}^{\text {loc }}\left(\mathbb{R}_{+}\right)$, и форма $\left(D_{t}\right)_{*}[\varphi]$ кусочно непрерьвна как функция параметра $t$ для любых $\varphi \in \operatorname{dom} \Lambda_{*}$. 
ЛЕмма 4.1. Если предположения (2.1), (2.4), (4.2) выполнены на каждом интервале $\left(t_{k-1}, t_{k}\right]$, то минимальное решение неоднородного уравнения Линдблада

$$
\frac{d}{d t} P_{t}=\mathscr{L}_{t}\left(P_{t}\right)+D_{t},\left.\quad P\right|_{t=0}=0
$$

допускает оценку

$$
\left(P_{t}^{\min }\right)_{*}[\varphi] \leqslant \Lambda_{*}[\varphi] \int_{0}^{t} d s \exp \left\{\int_{s}^{t} c_{\Lambda}(r) d r\right\} c_{D}(s) \quad \forall \varphi \in \operatorname{dom} \Lambda_{*} .
$$

ДокАЗАТЕЛЬСтво. В этом случае формула Дюамеля имеет вид

$$
\begin{aligned}
\left(P_{t}^{\min }\right)_{*}[\varphi] & =\int_{0}^{t} d s P_{t-t_{n}}^{(n)}\left(\cdots P_{t_{k(s)}-s}^{(k(s))}\left(D_{s}\right)\right)_{*}[\varphi]=\int_{0}^{t} d s \operatorname{Tr}\left\{T_{t_{k(s)}-s}^{(k(s))} \cdots T_{t-t_{n}}^{(n)}\left(\rho_{\varphi}\right) D_{s}\right\} \\
& \leqslant \int_{0}^{t} d s \operatorname{Tr}\left\{T_{t_{k(s)}-s}^{(k(s))} \cdots T_{t-t_{n}}^{(n)}\left(\rho_{\varphi}\right) \Lambda\right\} c_{D}(s)
\end{aligned}
$$

где $T_{t}^{(k)}(\cdot)$ - полугруппа, преддвойственная $P_{t}^{(k)}(\cdot)$, действующая в $\mathscr{T}(\mathscr{H}), \rho_{\varphi}=$ $|\varphi\rangle\langle\varphi| \in T_{\Lambda}(\mathscr{H})$. Поэтому из теоремы 3.1 следует оценка

$$
\begin{aligned}
\left(P_{t}^{\min }\right)_{*}[\varphi] & \leqslant \operatorname{Tr}\left(\rho_{\varphi} \Lambda\right) \int_{0}^{t} d s e^{\left(t-t_{n}\right) c_{\Lambda}\left(t_{n}\right)+\cdots+\left(t_{k(s)}-s\right) c_{\Lambda}\left(t_{k-1}\right)} c_{D}(s) \\
& =\Lambda_{*}[\varphi] \int_{0}^{t} d s \exp \left\{\int_{s}^{t} c_{\Lambda}(r) d r\right\} c_{D}(s) \quad \forall \varphi \in \mathscr{D} .
\end{aligned}
$$

Лемма доказана.

Пусть теперь $\mathscr{L}_{t}(\cdot)$ - функция параметра $t$, непрерьвная в том смысле, что для любого $\rho \in \mathscr{T}_{\Lambda}^{+}(\mathscr{H})$ функция

$$
\delta_{s, t}(B, \rho)=\left|\operatorname{Tr}\left\{\rho\left(\mathscr{L}_{t}(B)-\mathscr{L}_{s}(B)\right)\right\}\right|=\left|\operatorname{Tr}\left\{B\left(\mathscr{L}_{t}^{\dagger}(\rho)-\mathscr{L}_{s}^{\dagger}(\rho)\right)\right\}\right| \rightarrow 0
$$

почти всюду при $|t-s| \rightarrow 0$ и

$$
\varlimsup_{s \rightarrow t} \sup _{\|B\| \leqslant 1}\left|\delta_{s, t}(B, \rho)\right| \leqslant K_{t} \operatorname{Tr}(\rho \Lambda)
$$

где $K_{t} \in \mathscr{L}_{1}^{\text {loc }}\left(\mathbb{R}_{+}\right)$.

Рассмотрим последовательность разбиений $\left\{t_{i}(N)\right\}$ полупрямой $R_{+}$такую, что $0 \leqslant t_{k(s)}(N)-s \leqslant 2^{-N}$ для любого $s \in \mathbb{R}_{+}$. Сопоставим инфинитезимальному оператору $\mathscr{L}_{t}(\cdot)$ последовательность простых инфинитезимальных операторов $\mathscr{L}_{\tau}^{(N)}(\cdot)=\mathscr{L}_{t_{k(\tau)}}(\cdot)$. Пусть $P_{t, s}^{(N)}-$ композиция минимальных решений уравнений с кусочно постоянньми генераторами $\mathscr{L}_{t}^{(N)}(\cdot)$. Рассмотрим разность $\delta_{t}^{(M, N)}=P_{t, 0}^{(N)}(B)-P_{t, 0}^{(M)}(B)$, которая равномерно ограничена и удовлетворяет неоднородному уравнению с тривиальным начальным условием

$$
\frac{d}{d t} \delta_{t}^{(M, N)}=\mathscr{L}_{t}^{(N)}\left(\delta_{t}^{(M, N)}\right)+D_{t}^{(M, N)},\left.\quad \delta_{t}^{(M, N)}\right|_{t=0}=0
$$


где $D_{t}^{(M, N)}=\mathscr{L}_{t}^{(N)}\left(P_{t, 0}^{(M)}(B)\right)-\mathscr{L}_{t}^{(M)}\left(P_{t, 0}^{(M)}(B)\right)$, т.е. $-2 K_{t} \operatorname{Tr}(\rho \Lambda) \leqslant \operatorname{Tr}\left\{\rho D_{t}^{(M, N)}\right\} \leqslant$ $2 K_{t} \operatorname{Tr}(\rho \Lambda)$. Поскольку разность $\delta_{t}^{(M, N)}$ ограничена по норме, достаточно доказать ультраслабую сходимость $\delta_{t}^{(M, N)} \rightarrow 0$ при $M, N \rightarrow \infty$ на произвольном плотном подмножестве в $\mathscr{T}_{\Lambda}(\mathscr{H})$. В качестве такого подмножества мы используем линейную оболочку множества чистых состояний $\rho_{\varphi}=|\varphi\rangle\langle\varphi|, \varphi \in \operatorname{dom} \Lambda_{*}$.

Лемма 4.1 гарантирует равномерную по $M, N$ оценку для $\operatorname{Tr}\left\{\rho_{\varphi} \delta_{t}^{(M, N)}\right\}$ :

$$
\begin{aligned}
\left|\operatorname{Tr}\left\{\rho_{\varphi} \delta_{t}^{(M, N)}\right\}\right| & =\left|\int_{0}^{t} d s \operatorname{Tr}\left\{T_{t_{k(s)}-s}^{(k(s))} \cdots T_{t-t_{n}}^{(n)}\left(\rho_{\varphi}\right) D_{s}^{(M, N)}\right\}\right| \\
& \leqslant \operatorname{Tr}\{\rho \Lambda\} \int_{0}^{t} d s \exp \left\{\int_{s}^{t} c_{\Lambda}(s) d \tau\right\} K_{s} .
\end{aligned}
$$

В силу существования равномерно ограниченной мажоранты из $\mathscr{L}_{1}^{\text {loc }}\left(\mathbb{R}_{+}\right)$для подьнтегрального выражения, можно использовать теорему Лебега о предельном переходе под знаком интеграла и предположение (4.3):

$$
\lim _{M, N \rightarrow \infty}\left|\operatorname{Tr}\left\{\rho \delta_{t}^{(M, N)}\right\}\right| \leqslant \int_{0}^{t} d s \exp \left\{\int_{s}^{t} c_{\Lambda}(\tau) d \tau\right\} \delta_{s, t_{k(s)}}(B, \rho) \rightarrow 0
$$

при $M, N \rightarrow \infty$, поскольку $s-t_{k(s)} \rightarrow 0$.

Окончательно получаем следуюший результат.

Теорема 4.1. Предположсения (2.1), (2.4), (4.3), (4.4) достаточны для того, чтобъ последовательность $\left\{P_{t, s}^{(N)}(B)\right\}$ бълла ультраслабо фундаментальной $\mathscr{H} и$ сходилась к консервативному вполне положительному коциклу $P_{t, s}(B)$.

Рассмотрим класс $\mathscr{S}_{\Lambda}$ простых инфинитезимальных отображений $\mathscr{L}_{t}(\cdot)$, удовлетворяющих предположениям теоремы 4.1.

ОПРедЕЛЕниЕ 4.1. Последовательность $\left\{\mathscr{L}_{t}^{(N)}(\cdot)\right\}$ из $\mathscr{I}_{\Lambda}$ назьвается $\operatorname{Tr}_{\Lambda}$ - $у$ нндаментальной, если она сходится в локально выпуклой топологии, порождаемой системой полунорм

$$
\sigma_{\rho, T}\left(\mathscr{L}_{t}^{(N)}\right)=\int_{0}^{T} d t \sup _{\|B\| \leqslant 1}\left|\operatorname{Tr}\left\{\rho \mathscr{L}_{t}^{(N)}(B)\right\}\right| \exp \left\{\int_{s}^{t} d \tau c_{\Lambda}(\tau)\right\},
$$

где $T>0, \rho \in \mathscr{T}_{\Lambda}^{+}(\mathscr{H})$. Инфинитезимальное отображение с переменньми коэффициентами $\mathscr{L}_{t}(\cdot)$ назьвается $S_{\Lambda}$-измеримым, если последовательность

$$
\mathscr{L}_{t}^{(N)}(\cdot)=\mathscr{L}_{t_{k}}(\cdot), \quad t \in\left(t_{k}, t_{k+1}\right], \quad t_{k}-t_{k-1}=2^{-N},
$$

сходится к $\mathscr{L}_{t}(\cdot)$ относительно полунорм $(4.5)$.

Теорема 4.1 показывает, что $S_{\Lambda}$-измеримые инфинитезимальные отображения образуют естественный класс генераторов консервативных марковских коциклов. 
5. ПримеР. Пусть $\mathscr{H}=L_{2}(\mathbb{R})$ и коэффициенты отображения $\mathscr{L}_{t}(\cdot)$ равны

$$
H_{t}=\Omega(x, t) x^{2}, \quad \Phi_{t}(B)=-\partial_{x} a(x, t) B a(x, t) \partial_{x},
$$

где $a$ и $\Omega$ - гладкие ограниченные функции такие, что

$$
\sup _{x} a(x, t)<\infty, \sup _{x}|\Omega(x, t)|<\infty, \inf _{x} a(x, t)>0 \quad \forall t \in[0, T] .
$$

Обозначим $A(x, t)=a^{2}(x, t)$. Оператор $L_{t}=\Phi_{t}(I), L_{t}=\frac{1}{2} A(x, t) \partial_{x}^{2}+a a_{x}^{\prime} \partial_{x}$, является производящим оператором диффузионного процесса $\xi_{\tau}$ (см. [11]), удовлетворяющего стохастическому дифференциальному уравнению

$$
d \xi_{\tau}=a\left(\xi_{\tau}, \tau\right) d w_{\tau}+a\left(\xi_{\tau}, \tau\right) a_{x}^{\prime}\left(\xi_{\tau}, \tau\right) d \tau, \quad \xi_{t}=x,
$$

где $w_{\tau}$ - стандартный винеровский процесс, а двухпараметрическое семейство сжимающих операторов $W_{s, t}$ с генератором $-G_{t}, G_{t}=\frac{1}{2} \Phi_{t}(I)+i H_{t}$, может быть представлено в виде условного математического ожидания

$$
W_{s, t} \psi(x)=\mathbb{M}_{x, t} \psi\left(\xi_{s}\right) \exp \left\{-i \int_{s}^{t} d \tau \Omega\left(\xi_{\tau}, \tau\right) \xi_{\tau}^{2}\right\} .
$$

Проверим достаточные условия консервативности (2.4) и критерий непрерьвности (4.3), (4.4) относительно оператора

$$
\Lambda=\lambda\left(-\partial_{x}^{2}+x^{2}+I\right), \quad \lambda=\sup _{x \in \mathbb{R}, t \in(0, T)}\{|\Omega(x, t)|+A(x, t)\} .
$$

Преждевсего, имеем тождество для коммутатора: $i\left[H_{t}, \Lambda\right]=i\left[\Omega x^{2}, \partial_{x}^{2}\right]=-i\left\{b \partial_{x}+\partial_{x} b\right\}$, где $b=\partial_{x}\left(\Omega(x, t) x^{2}\right)$. Из известного неравенства

$$
\pm\left(A^{*} B+B^{*} A\right) \leqslant A^{*} \varepsilon A+B^{*} \varepsilon^{-1} B,
$$

справедливого для любого $\varepsilon \in \mathbb{R}_{+}$, в частном случае $A=i \partial_{x}, B=b=\partial_{x}\left(\Omega x^{2}\right), \varepsilon=1$ имеем

$$
i\left[H_{t}, \Lambda\right] \leqslant\left(b^{2}-\partial_{x}^{2}\right) \leqslant \lambda \Lambda, \quad \text { если } \sup _{t \in(0, T)}(1+|x|)^{-1}\left|\partial_{x} \Omega x^{2}\right| \leqslant \lambda .
$$

Непосредственные вычисления показывают, что

$$
\begin{aligned}
& \Phi_{t}\left(x^{2}\right)-\Phi_{t}(I) \circ x^{2}=2 a(x, t) a_{x}^{\prime}(x, t) x+a^{2}(x, t), \\
& \Phi_{t}\left(\partial_{x}^{2}\right)-\Phi_{t}(I) \circ \partial_{x}^{2}=-\left(a_{x}^{\prime}(x, t)\right)^{2} .
\end{aligned}
$$

Поэтому условие (2.4) выполнено, если

$$
\sup _{x, t}(1+|x|)\left|\Omega_{x}^{\prime}(x, t)\right|<\infty, \quad \sup _{x, t}(1+|x|)^{-1}\left|a_{x}^{\prime}(x, t)\right|<\infty .
$$

Проверим условие непрерьвности. Пусть $\rho_{\varphi} \in \mathscr{T}\left(\mathscr{H}_{\Lambda}\right)$. Учитьвая, что $\left|\operatorname{Tr} A_{n} \rho\right| \rightarrow 0$, если $\sup _{n}\left\|A_{n}\right\|<\infty$, и $A_{n} \rightarrow 0$ в сильном смысле, имеем

$$
\operatorname{Tr}\left\{B\left[H_{t}-H_{s}, \rho\right]\right\}=\operatorname{Tr}\left\{(\Omega(x, t)-\Omega(x, s)) x^{2} \rho\right\} \rightarrow 0,
$$


если почти всюду в $\mathbb{R}$

$$
|\Omega(x, t)-\Omega(x, s)| \rightarrow 0
$$

сходится к нулю при $t-s \rightarrow 0$. Далее, из неравенства (5.1) вытекает оценка для разности вполне положительных отображений

$$
\Phi_{t}^{\dagger}(\rho)-\Phi_{s}^{\dagger}(\rho) \leqslant \frac{\varepsilon}{2}\left(\Phi_{t}^{\dagger}(\rho)+\Phi_{s}^{\dagger}(\rho)\right)+\varepsilon^{-1}(a(x, t)-a(x, s)) \partial_{x} \rho \partial_{x}(a(x, t)-a(x, s)) .
$$

Поэтому

$$
\left|\operatorname{Tr}\left(B\left(\Phi_{t}^{\dagger}(\rho)-\Phi_{s}^{\dagger}(\rho)\right)\right)\right| \leqslant \lambda \varepsilon\|B\| \operatorname{Tr}(\rho \Lambda)+\varepsilon^{-1} \operatorname{Tr}\left\{(a(\cdot, t)-a(\cdot, s))^{2} \rho \Lambda\right\} \rightarrow 0,
$$

если $|a(x, t)-a(x, s)| \rightarrow 0$ при $t-s \rightarrow 0$ для почти всех $x \in \mathbb{R}$. Аналогично доказьвается неравенство

$$
\operatorname{Tr}\left\{B \rho\left(\Phi_{s}^{\dagger}(I)-\Phi_{t}^{\dagger}(I)\right) \circ \rho\right\} \leqslant\|B\| \operatorname{Tr}\{\rho \Lambda \varphi(s, t)\},
$$

где $\varphi(s, t)=\left(\Phi_{s}^{\dagger}(I)-\Phi_{t}^{\dagger}(I)\right) \Lambda^{-1}$.

Нетрудно видеть, что семейство операторов $\varphi(s, t)$ равномерно ограничено. Заметим, далее, что $a \partial_{x}^{2}=\partial_{x}^{2} a-\left(\partial_{x} a_{x}^{\prime}+a_{x}^{\prime} \partial_{x}\right)$ и поэтому

$$
\|\varphi(s, t) \psi\| \leqslant\left\|\left(\left|a^{2}(\cdot, t)-a^{2}(\cdot, s)\right|+\varepsilon^{-1}\left|a_{x}^{\prime}(\cdot, t)-a_{x}^{\prime}(\cdot, s)\right|^{2}\right) \partial_{x}^{2} \Lambda^{-1} \psi\right\|+\varepsilon\left\|\partial_{x}^{2} \Lambda^{-1} \psi\right\|
$$

для сколь угодно малого $\varepsilon$. Таким образом, $\|\varphi(s, t) \psi\| \rightarrow 0$, если при $t-s \rightarrow 0$ для почти всех $x \in \mathbb{R}$ имеет место сходимость

$$
|a(\cdot, t)-a(\cdot, s)| \rightarrow 0, \quad(1+|x|)^{-1}\left|a_{x}^{\prime}(\cdot, t)-a_{x}^{\prime}(\cdot, s)\right| \rightarrow 0 .
$$

Следовательно, предельньй марковский коцикл $P_{t}(\cdot)=w^{*}-\lim P_{t}^{(N)}(\cdot)$ существует и консервативен, если для равномерно ограниченных коэффициентов $a(x, t), \Omega(x, t)$ выполнены условия непрерывности (5.2), (5.3), (5.4).

Московский государственный университет им. М.В. Ломоносова

Depto. de Matemáticas, UAM-I, Av. Michoacán y la Purísima (México)

27.11 .95

E-mail: alex@cheb.phys.msu.su

\section{СПИСОК ЦИТИРОВАННОЙ ЛИТЕРАТУРЫ}

[1] Davies E. B. Quantum theory of open systems. London: Acad. Press, 1976.

[2] Gorini V., Kossakovsky A., Sudarshan E. C. G. Completely positive dynamical semigroups of $n$-level systems // J. Math. Phys. 1976. V. 17. №3. P. 821-825.

[3] Lindblad G. On the generators of quantum dynamical semigroups // Commun. Math. Phys. 1976. V. 48. № 2. P. 119-130.

[4] Чеботарев А. М. Необходимое и достаточное условие консервативности динамической полугруппы // Итоги науки и техн. Соврем. пробл. матем. Новейшие достижения. Т. 36. М.: ВИНИТИ, 1990. С. 149-184.

[5] Chebotarev A. M., Fagnola F. Sufficient conditions for conservativity of quantum dynamical semigroup // J. Funct. Anal. 1993. V. 118. №1. P. 131-153.

[6] Чеботарев А. М. Достаточное условие консервативности минимальной динамической полугруппы // Матем. заметки. 1992. Т. 52. № 4. С. 112-122.

[7] Chebotarev A. M. Applications of quantum probability to classical stochastics. Preprint. V. 246: Centro V. Volterra, Univ. degli studi di Roma "Tor Vergata", 1996.

[8] Chebotarev A. M., Fagnola F. Sufficient conditions for conservativity of minimal quantum dynamical semigroups // J. Funct. Anal. (to appear).

[9] Браттели У., Робинсон Д. Операторные алгебры и квантовая статистическая механика. M.: Мир, 1982.

[10] Като Т. Теория возмущений линейных операторов. М.: Наука, 1972.

[11] McKean G. Stochastic integrals. Princeton: Univ. Press, 1981. 